\title{
Clinical Significance of Arterial Velocity Pulse Index in Patients With Stage B Heart Failure With Preserved Ejection Fraction
}

\author{
Takashi Hitsumoto
}

\begin{abstract}
Background: In clinical settings, the arterial velocity pulse index (AVI) is explored as a novel marker of atherosclerosis using pulse wave analysis; however, data regarding the correlations between AVI and heart failure (HF) are limited. This study aimed to elucidate the clinical significance of AVI in patients with stage B HF with preserved ejection fraction (HFpEF).

Methods: In this cross-sectional study, 345 patients with stage B HFpEF (no symptoms despite evidence of cardiac structural or functional impairment, and left ventricular ejection fraction which is estimated by echocardiography $\geq 50 \%$ ) were enrolled. Patients with a history of HF hospitalization were excluded. The AVI was measured using a commercial device, and associations between AVI and various clinical parameters were examined.
\end{abstract}

Results: Significant correlations between AVI and various clinical parameters, such as E/e' as a maker of left ventricular diastolic function $(\mathrm{r}=0.35 ; \mathrm{P}<0.001)$, high-sensitivity cardiac troponin $\mathrm{T}$ levels as a marker of myocardial injury $(\mathrm{r}=0.47 ; \mathrm{P}<0.001)$, reactive oxygen metabolite levels as an oxidative stress marker $(\mathrm{r}=0.31 ; \mathrm{P}<0.001)$, urinary albumin concentration as a marker of kidney function $(\mathrm{r}=$ $0.34 ; \mathrm{P}<0.001)$ and calf circumference as a marker of muscle mass volume $(\mathrm{r}=-0.42 ; \mathrm{P}<0.001)$ were observed. Furthermore, multiple regression analyses revealed that these clinical parameters were selected as independent variables when AVI was used as a subordinate factor.

Conclusions: This study shows that AVI might be a determining factor for prognosis in patients with stage B HFpEF. Nevertheless, further comprehensive prospective studies, including intervention therapies, are warranted to validate the findings of this study.

Keywords: Arterial velocity pulse index; Heart failure with preserved ejection fraction; Stage B; Left ventricular diastolic function; High-sensitivity cardiac troponin T; Oxidative stress; Sarcopenia

Manuscript submitted April 9, 2019, accepted April 17, 2019

Hitsumoto Medical Clinic, 2-7-7, Takezakicyou, Shimonoseki City, Yamaguchi 750-0025, Japan. Email: thitsu@jcom.home.ne.jp

doi: https://doi.org/10.14740/cr864

\section{Introduction}

In recent years, extended life expectancy or lifestyle changes have markedly contributed to the elevated incidence of heart failure (HF) worldwide. Contrarily, there have been remarkable advancements with multiple therapeutic agents of proven benefit in HF with reduced ejection fraction; HF with preserved ejection fraction (HFpEF) lacks evidence-based therapies [1,2]. In addition, epidemiological studies have reported that patients with HF with a history of HF hospitalization exhibit poor prognosis because of rehospitalization owing to HF worsening, cardiovascular diseases, or other adverse events $[3,4]$. Thus, it is imperative to investigate the novel diagnosis and therapy for HFpEF before the prevalence of HF hospitalization or symptoms of HF such as dyspnea or edema.

The arterial velocity pulse index (AVI), a novel marker of atherosclerosis, uses oscillometric cuffs to measure pulse waveforms [5]. Increments in AVI depict the enhancement of reflected waves resulting from atherosclerosis or other parameters. In addition, several clinical studies reported the clinical efficacy of AVI as a cardiovascular risk factor [6-8]. Conversely, some studies reported that physiological markers of atherosclerosis, such as pulse wave velocity, cardio-ankle vascular index, augmentation index (AIx) and flow-mediated dilation, correlated with the pathogenesis or prognosis of HF [9-12]. Nevertheless, data regarding the correlations between AVI and HF, including HFpEF, are limited. Hence, this cross-sectional study aimed to elucidate the clinical significance of AVI in patients with stage B HFpEF.

\section{Materials and Methods}

\section{Patients}

This cross-sectional study was performed at the Hitsumoto Medical Clinic in Yamaguchi, Japan, between December 2015 and November 2018. A total of 345 patients with stage B HFpEF (no symptoms despite evidence of cardiac structural or functional impairment, and left ventricular ejection fraction which is estimated by echocardiography $\geq 50 \%$ ) were enrolled. Stage B HF was defined based on the ACC/AHA 2005 Guidelines for the Diagnosis and Management of Heart 


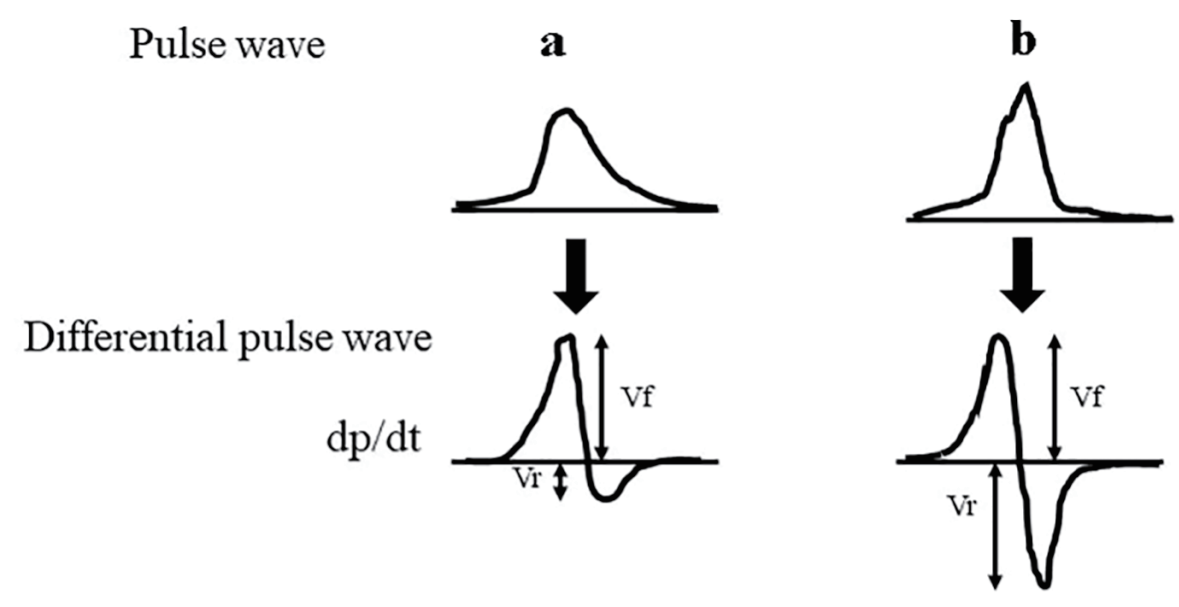

$\mathrm{AVI}=(\mathrm{Vr} / \mathrm{Vf}) \times 20$

Figure 1. The measurement concept of the AVI. AVI has the characteristics of a pulse wave pattern at higher cuff pressures than the systolic blood pressure. We measured two variables automatically, i.e. Vf (the first peak of the differential of the waveform with respect to time) and $\mathrm{Vr}$ (the absolute value of the bottom of the valley of the differential of the waveform with respect to time), and AVI was automatically calculated as $20 \times(\mathrm{Vr} / \mathrm{Vf})$. (a) Pulse wave pattern of low AVI levels. (b) Pulse wave pattern of high AVI levels. AVI: arterial velocity pulse index.

Failure in Adults [13]. Of note, patients with a history of HF hospitalization were excluded from the study. Patients comprised 121 males $(35.0 \%)$ and 224 females $(65.0 \%)$, with the mean age of $74 \pm 7$ years (mean \pm standard deviation). This study was conducted in compliance with the ethical standards of the responsible institution on human subjects as well as the Helsinki Declaration. The study protocol was approved by the Institutional Review Board of the Hitsumoto Medical Clinic (approval number: 2015-11).

\section{AVI measurement}

The AVI was measured using a commercially available instrument (PASSESA AVI-1500; Shisei Datum, Tokyo, Japan) in the sitting position as previously described [5]. All measurements were performed when participants were in a quiet room (temperature, $20-25^{\circ} \mathrm{C}$ ). Figure 1 shows the measurement concept of AVI. Of note, AVI has the characteristics of a pulse wave pattern at higher cuff pressures than the systolic blood pressure. We measured two variables automatically, i.e. Vf (the first peak of the differential of the waveform with respect to time) and $\mathrm{Vr}$ (the absolute value of the bottom of the valley of the differential of the waveform with respect to time), and AVI was automatically calculated as $20 \times(\mathrm{Vr} / \mathrm{Vf})$. Although the systolic waveform rapidly increases and decreases in response to reflected arterial waves, the initial waveform is not affected by the reflected arterial waves. Vf indicates the initial waveform, while Vr indicates the subsequent (weaker) waveform affected by reflected arterial waves. As the wave reflections become stronger, AVI increases in parallel with an increase in Vr. Prior studies have established the validity and reliability of AVI measured using this method $[14,15]$.

\section{Assessment of clinical parameters}

Various clinical parameters such as physical measurement, traditional cardiovascular risk factor, history of ischemic heart disease, blood glucose-related parameters, echocardiographic findings, kidney function, brain natriuretic peptide (BNP) levels, high-sensitivity cardiac troponin $\mathrm{T}$ (hs-cTnT) levels and oxidative stress were evaluated. Obesity was defined using the body mass index (BMI), evaluated as the weight $(\mathrm{kg})$ divided by the squared height $\left(\mathrm{m}^{2}\right)$. Calf circumferance was mesured as a surrogate marker of muscle mass volume [16]. Current smoking was defined as smoking, at least, one cigarette per day over the previous 28 days. In addition, history of ischemic heart disease was defined as patients with a history of myocardial infarction and/or angiography-proven significant stenosis. Hypertension was defined as systolic blood pressure $\geq 140$ $\mathrm{mm} \mathrm{Hg}$, diastolic blood pressure $\geq 90 \mathrm{~mm} \mathrm{Hg}$, or those taking antihypertensive medications. Dyslipidemia was defined as low-density lipoprotein cholesterol levels $\geq 140 \mathrm{mg} / \mathrm{dL}$, highdensity lipoprotein cholesterol levels $\leq 40 \mathrm{mg} / \mathrm{dL}$, triglyceride levels $\geq 150 \mathrm{mg} / \mathrm{dL}$, or based on ongoing treatment for dyslipidemia. We defined diabetes mellitus as having fasting blood glucose levels of $\geq 126 \mathrm{mg} / \mathrm{dL}$ or hemoglobin A1c levels of $\geq$ $6.5 \%$, which is estimated by the National Glycohemoglobin Standardization Program, or taking of antidiabetic treatment. We measured glucose and insulin levels using the glucose oxidase method and an enzyme immunoassay, respectively. To measure insulin resistance, HOMA-IR was calculated using the following equation [17]: HOMA-IR = fasting glucose concentration $(\mathrm{mg} / \mathrm{dL}) \times$ fasting insulin concentration $(\mu \mathrm{g} /$ 
$\mathrm{mL}) / 405$. The standard technique for echocardiography was performed using HI VISION Avius (Hitachi Medical Corporation, Tokyo, Japan), and valvular heart disease was diagnosed on the basis of the Japanese Circulation Society guidelines (Guidelines for the Clinical Application of Echocardiography (JCS 2010)). Valvular heart disease comprised aortic or mitral valve disease (aortic stenosis, aortic regurgitation, mitral stenosis and mitral regurgitation). Using echocardiography, left ventricular wall thickness, left ventricular extended period diameter, left ventricular ejection fraction, left atrial dimension and $\mathrm{E} / \mathrm{e}^{\prime}$ were measured. The estimated glomerular filtration rate (eGFR) was evaluated using the adjusted Modification of Diet in the Renal Disease Study equation, which was proposed by the working group of the Japanese Chronic Kidney Disease Initiative [18]. The urinary albumin concentration was measured using a commercial kit (Siemens/Bayer DCA 2000+ analyzer; Siemens Healthineers, Tokyo, Japan). Moreover, we measured BNP levels using a commercial kit (SHIONOSPOT Reader; Shionogi \& Co., Osaka, Japan) and hs-cTnT levels using a commercial kit (Roche Diagnostics, Basel, Switzerland) [19]. As an oxidative stress maker in vivo [20], the reactive oxygen metabolites (d-ROMs) test was performed using a commercial kit (Diacron, Grosseto, Italy).

\section{Statistical analysis}

In this study, data were analyzed using MedCalc for Windows (version 14.8.1; MedCalc Software, Ostend, Belgium) and StatView J5.0 (HULINKS, Tokyo, Japan). Data are presented as mean \pm standard deviation. The correlation coefficient was estimated by Spearman rank order correlation analysis. In addition, the multivariate analysis was conducted by multiple regression analysis. The receiver-operating characteristic (ROC) curves were constructed, and the maximum Youden index [21] was used to determine the optimal AVI cutoff levels for determining high E/e', high hs-cTnT and low calf circumference. Furthermore, we considered $\mathrm{P}<0.05$ as statistically significant.

\section{Results}

Table 1 summarizes the patients' characteristics. The mean AVI levels were $30 \pm 7$ (range: 14 - 54). The distribution of AVI exhibited nearly normal distribution. Table 2 presents the correlations between AVI and various clinical parameters. In addition, age, presence of diabetes mellitus, fasting blood glucose levels, left ventricular wall thickness, E/e', urinary albumin concentration, BNP, hs-cTnT and d-ROMs test exhibited significantly positive correlation with AVI. However, male gender, body height, BMI, pulse rate, eGFR, renin-angiotensin system inhibitor use and statin use exhibited significantly negative correlation with AVI. Table 3 summarizes the results of a multiple regression analysis with AVI as a subordinate factor. Explanatory factors were selected by examining multicollinearity among the variables or by conducting a stepwise method, and nine factors were selected. Of those nine factors, seven factors (E/e', hs-cTnT, calf circumference, urinary albu-
Table 1. Patient Characteristics

\begin{tabular}{|c|c|}
\hline n (male/female) & $345(121 / 224)$ \\
\hline Age (years) & $74 \pm 7$ \\
\hline Body height (cm) & $155 \pm 10$ \\
\hline Body mass index (kg/m2) & $22.8 \pm 3.8$ \\
\hline Calf circumference $(\mathrm{cm})$ & $32.5 \pm 3.0$ \\
\hline Current smoker, n (\%) & $56(16)$ \\
\hline Ischemic heart disease, $\mathrm{n}(\%)$ & $85(25)$ \\
\hline Hypertension, n (\%) & $256(74)$ \\
\hline Systolic blood pressure (mm Hg) & $143 \pm 22$ \\
\hline Diastolic blood pressure ( $\mathrm{mm} \mathrm{Hg}$ ) & $82 \pm 10$ \\
\hline Pulse rate (/min) & $70 \pm 11$ \\
\hline Dyslipidemia, n (\%) & $227(66)$ \\
\hline Diabetes mellitus, n (\%) & $124(36)$ \\
\hline Fasting blood glucose (mg/dL) & $109 \pm 28$ \\
\hline HOMA-IR & $2.1 \pm 1.7$ \\
\hline Hemoglobin A1c (\%) & $6.2 \pm 1.0$ \\
\hline Heart valvular disease, $\mathrm{n}(\%)$ & $269(78)$ \\
\hline IVSTd (mm) & $9.8 \pm 1.7$ \\
\hline LVDd (mm) & $50.4 \pm 3.6$ \\
\hline LVEF (\%) & $67.7 \pm 8.7$ \\
\hline $\mathrm{LAD}(\mathrm{mm})$ & $42.6 \pm 5.6$ \\
\hline $\mathrm{E} / \mathrm{e}^{\prime}$ & $10.5 \pm 3.3$ \\
\hline $\mathrm{eGFR}\left(\mathrm{mL} / \mathrm{min} / 1.73 \mathrm{~m}^{2}\right)$ & $49.9 \pm 18.9$ \\
\hline Log-urinary albumin (mg/g Cr) & $1.8 \pm 0.5$ \\
\hline $\log -\mathrm{BNP}(\mathrm{pg} / \mathrm{mL})$ & $2.0 \pm 0.3$ \\
\hline Log-hs-cTnT (ng/mL) & $-2.0 \pm 0.3$ \\
\hline d-ROMs test (U. CARR) & $304 \pm 113$ \\
\hline AVI & $30 \pm 7$ \\
\hline \multicolumn{2}{|l|}{ Medication } \\
\hline RAS inhibitor, n (\%) & $217(63)$ \\
\hline$\beta$ blocker, n (\%) & $71(21)$ \\
\hline Diuretics, n (\%) & $70(20)$ \\
\hline Statin, n (\%) & $130(38)$ \\
\hline
\end{tabular}

Continuous values are mean ${ }_{ \pm}$SD. HOMA-IR: homeostasis assessment insulin resistance; IVSTd: interventricular septal thickness at enddiastole; LVDd: left ventricular end-diastolic diameter; LVEF: left ventricular ejection fraction; LAD: left atrial dimension; eGFR: estimated glomerular filtration rate; BNP: brain natriuretic peptide; hs-cTnT: highsensitivity cardiac troponin $\mathrm{T}$; d-ROMs: derivatives of reactive oxygen metabolites; AVI: arterial velocity pulse index; RAS: renin-angiotensin system.

min concentration, body height, d-ROM test and pulse rate) were identified as independent variables when AVI was used as a subordinate factor. Figure 2 shows the ROC curve analysis for the detection of high E/e' of $>15$, high hs-cTnT of $\geq 0.014$ $\mathrm{ng} / \mathrm{mL}$ and low calf circumference $(<34 \mathrm{~cm}$ in males and $<$ $33 \mathrm{~cm}$ in females) based on previous studies. The maximum 
Table 2. AVI and Various Clinical Parameters

\begin{tabular}{|c|c|c|}
\hline & $\mathbf{r}$ & Pvalue \\
\hline Sex $($ female $=0$, male $=1)$ & -0.16 & 0.002 \\
\hline Age & 0.18 & $<0.001$ \\
\hline Body height & -0.20 & $<0.001$ \\
\hline Body mass index & -0.18 & $<0.001$ \\
\hline Calf circumference & -0.42 & $<0.001$ \\
\hline Current smoker $($ no $=0$, yes $=1)$ & 0.10 & 0.072 \\
\hline Ischemic heart disease $($ no $=0$, yes $=1)$ & 0.08 & 0.160 \\
\hline Hypertension $($ no $=0$, yes $=1)$ & 0.02 & 0.684 \\
\hline Systolic blood pressure & 0.03 & 0.539 \\
\hline Diastolic blood pressure & 0.02 & 0.730 \\
\hline Pulse rate & -0.20 & $<0.001$ \\
\hline Dyslipidemia $($ no $=0$, yes $=1)$ & 0.09 & 0.078 \\
\hline Diabetes mellitus (no $=0$, yes $=1$ ) & 0.17 & 0.002 \\
\hline Fasting blood glucose & 0.14 & 0.009 \\
\hline HOMA-IR & 0.04 & 0.481 \\
\hline Hemoglobin A1c & 0.09 & 0.105 \\
\hline Heart valvular disease & 0.08 & 0.153 \\
\hline IVSTd & 0.11 & 0.043 \\
\hline LVDd & 0.05 & 0.401 \\
\hline LVEF & 0.03 & 0.614 \\
\hline LAD & 0.07 & 0.183 \\
\hline E/e' & 0.35 & $<0.001$ \\
\hline eGFR & -0.12 & 0.024 \\
\hline Log-urinary albumin & 0.34 & $<0.001$ \\
\hline Log-BNP & 0.23 & $<0.001$ \\
\hline Log-hs-cTnT & 0.47 & $<0.001$ \\
\hline d-ROMs test & 0.31 & $<0.001$ \\
\hline RAS inhibitor $($ no $=0$, yes $=1)$ & -0.11 & 0.034 \\
\hline$\beta$ blocker $($ no $=0$, yes $=1)$ & 0.08 & 0.095 \\
\hline Diuretics $($ no $=0$, yes $=1)$ & 0.03 & 0.527 \\
\hline Statin $($ no $=0$, yes $=1)$ & -0.11 & 0.044 \\
\hline
\end{tabular}

$r$ expressed correlation coefficient. HOMA-IR: homeostasis assessment insulin resistance; IVSTd: interventricular septal thickness at enddiastole; LVDd: left ventricular end-diastolic diameter; LVEF: left ventricular ejection fraction; LAD: left atrial dimension; eGFR: estimated glomerular filtration rate; BNP: brain natriuretic peptide; hs-cTnT: high sensitivity cardiac troponin $\mathrm{T}$; $\mathrm{d}$-ROMs: derivatives of reactive oxygen metabolites; RAS: renin-angiotensin system.

Youden's index suggested that AVI of $>30$ was the optimal cutoff point to determine the high E/e', high hs-cTnT and low calf circumference.

\section{Discussion}

Previous studies demonstrated a significant correlation be-
Table 3. Multiple Regression Analysis for AVI

\begin{tabular}{lll}
\hline Explanatory factor & $\boldsymbol{\beta}$ & P value \\
\hline E/e' & 0.29 & $<0.001$ \\
Log-hs-cTnT & 0.27 & $<0.001$ \\
Calf circumference & -0.26 & $<0.001$ \\
Log-urinary albumin & 0.16 & $<0.001$ \\
Body height & -0.12 & 0.005 \\
d-ROMs test & 0.11 & 0.022 \\
Pulse rate & -0.10 & 0.024 \\
Sex (male) & -0.07 & 0.125 \\
Log-BNP & 0.06 & 0.189 \\
\hline
\end{tabular}

$R^{2}=0.41$. AVI: arterial velocity pulse index; hs-cTnT: high-sensitivity cardiac troponin $\mathrm{T}$; $\mathrm{d}$-ROMs: derivatives of reactive oxygen metabolites; BNP: brain natriuretic peptide; $\beta$ : standardized regression coefficient; $R^{2}$ : coefficient of determination.

tween the arterial reflection wave and body height or pulse rate $[22,23]$. Likewise, this study demonstrated that the body height and pulse rate were selected as the independent variables for AVI as a subordinate factor, suggesting that AVI depicts features of the arterial reflection wave. Conversely, E/e' as a maker of left ventricular diastolic function, hs-cTnT levels as a marker of myocardial injury, urinary albumin cancentration as a marker of kidney function, d-ROMs tests as an oxidative stress marker and calf circumference as a marker of muscle mass volume were selected as independent variables for AVI as a subordinate factor.

Left ventricular diastolic dysfunction plays a vital role in the HFpEF pathogenesis, and left ventricular diastolic dysfunction is reportedly caused by not only heart condition, such as left ventricular hypertrophy or myocardial fibrosis, but also arterial dysfunction [24, 25]. In addition, some clinical studies reported that the augmentation index as a representative marker of the arterial reflection wave exhibited a significant correlation with parameters of left ventricular diastolic dysfunction [26, 27]. Hence, significant correlations between AVI and $\mathrm{E} / \mathrm{e}^{\prime}$ in this study considered the significance of an increase in the arterial reflection wave, which is caused by various factors, including atherosclerosis for the diastolic function of stage B HFpEF. Meanwhile, hs-cTnT is used as a biomarker to assess the degree of myocardial injury in the clinical setting. Moreover, several studies have suggested the clinical efficacy of hs-cTnT as a prognostic value such as the incidence of allcause mortality or rehospitalization for $\mathrm{HF}$ in patients with HFpEF [28]. Hence, the significant correlation between AVI and hs-cTnT in this study could be interpreted that an increase in the arterial reflection wave caused myocardial damage; furthermore, AVI could provide prognosis in patients with stage B HFpEF.

Having a target value of AVI to prevent adverse cardiovascular events in patients with stage B HFpEF is beneficial. This study attempts to decide cutoff levels of AVI for detecting high E/e' of $>15$ or high hs-cTnT of $\geq 0.014 \mathrm{ng} / \mathrm{mL}$, which reportedly correlate with diastolic dysfunction, HF hospitalization and cardiovascular risk [29-31]. The ROC curve analysis for 

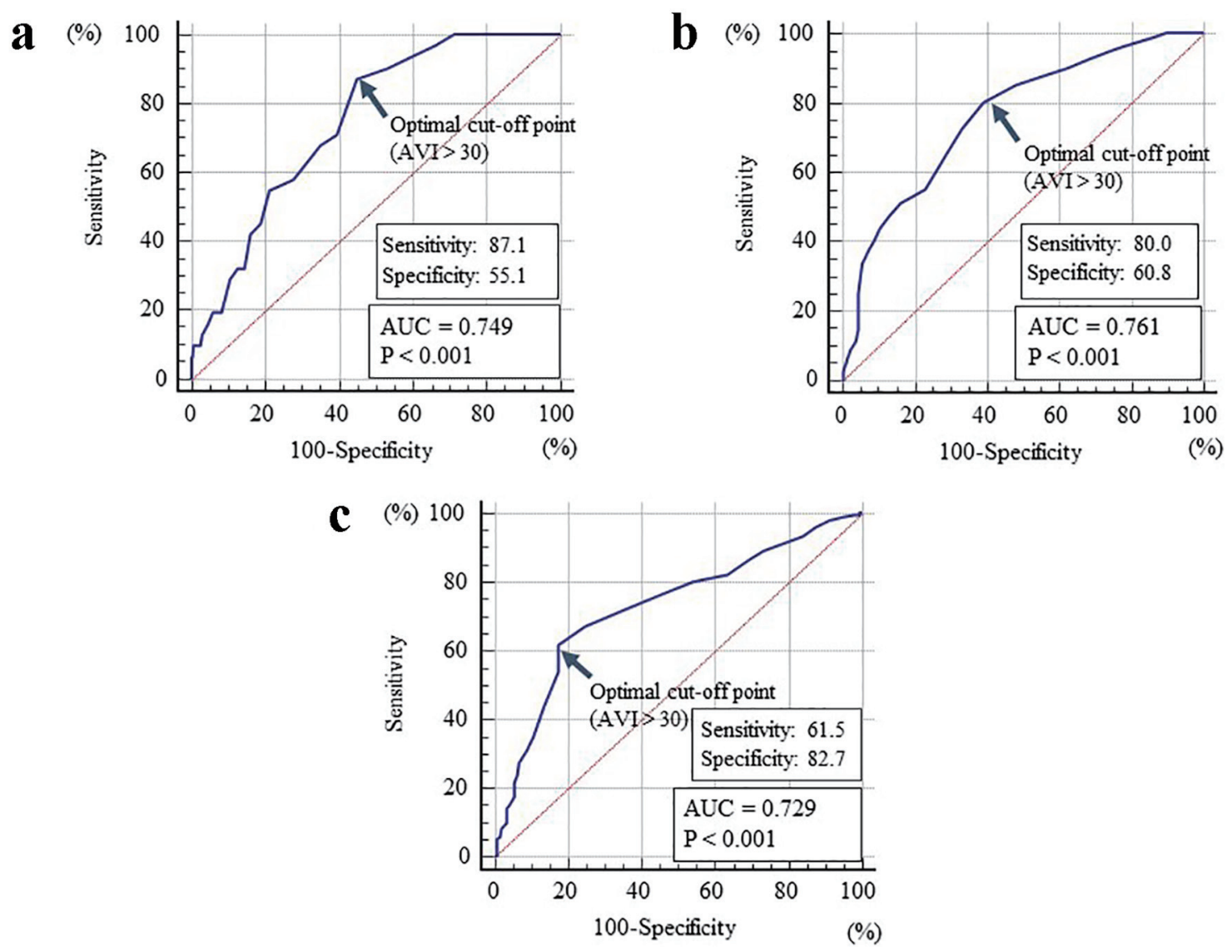

Figure 2. The cutoff value of the AVI for determining high E/e', high hs-cTnT and low calf circumference. (a) High E/e' of > 15; (b) high hs-cTnT of $\geq 0.014 \mathrm{ng} / \mathrm{mL}$; (c) low calf circumference of $<34 \mathrm{~cm}$ in males and $<33 \mathrm{~cm}$ in females. AVl: arterial velocity pulse index; hs-cTnT: high-sensitivity cardiac troponin T; AUC: area under the curve.

AVI as high of $>30$ suggested an optimal cutoff point for differentiating both high E/e' and hs-cTnT. Although the specificity is relatively low, this study indicates that the incidence of cardiovascular events, including initial HF hospitalization, in patients with stage B HFpEF could be prevented by maintaining AVI of $\leq 30$ in daily practice.

The urinary albumin concentration is a maker of not only kidney function but also systemic endothelial function [32]. Moreover, studies reported the significance of endothelial dysfunction in the HFpEF pathogenesis [33-35]. Contrarily, the PROMIS-HFpEF study demonstrated that a high incidence of coronary microvascular dysfunction in HFpEF correlates with systemic endothelial dysfunction, including urinary albumin concentration, and the authors deduced that microvascular dysfunction might be a promising therapeutic target in HFpEF [35]. Hence, an independent correlation between AVI and urinary albumin concentration suggested that AVI could signify not only systemic endothelial function but also coronary microvascular function; consequently, it is a useful target factor to prevent cardiovascular adverse events in patients with stage B HFpEF.

Some studies suggested close correlations between oxidative stress and HFpEF [36, 37]. In addition, oxidative stress is reportedly affected by an increase in the arterial reflection wave [38, 39]. Likewise, this study suggested a significant correlation between the d-ROMs test as a marker of oxidative stress and AVI. Contrarily, medications, such as renin-angiotensin system inhibitor and statins, exhibited antioxidative stress efficacy. In addition, several clinical studies reported that these medications exhibited positive effects on the arterial reflection wave [40, 41]. In this study, the renin-angiotensin system inhibitor and statin use exhibited significantly negative correlations with AVI in the univariate analysis, although these medications were not selected as variables for the multivariate analysis. Corroborating the literature, this study suggested that anti-oxidant medications, such as renin-angiotensin system inhibitor or statin, should be considered for patients with high AVI.

In recent years, several clinical studies have highlighted the significance of sarcopenia in the HFpEF pathogenesis [42]. In addition, other clinical studies reported that sarcopenia could be affected by an increase in the arterial reflection wave $[43,44]$. However, Kawakami et al reported that the calf circumference positively correlated with the appendicular skeletal muscle mass and skeletal muscle index, and could be used as a surrogate marker of the muscle mass for 
diagnosing sarcopenia [16]. Hence, an independent correlation between AVI and calf circumference could suggest that sarcopenia affected an increase in the arterial reflection wave; this caused increased left ventricular afterload, which, in turn, caused adverse cardiac events in patients with stage B HFpEF. Similarly, Kawakami et al reported that the cutoff values of calf circumference for predicting sarcopenia were $<34 \mathrm{~cm}$ in males and $<33 \mathrm{~cm}$ in females in the Japanese population. Conversely, AVI of $>30 \mathrm{~cm}$ was the optimal cutoff level for determining the low calf circumference, which was elucidated by Kawakami's report. Moreover, AVI of $>30 \mathrm{~cm}$ suggested a definitive value to determine the high E/e' or high hs-cTnT in this study. However, studies suggested that exercise training exhibited augmented calf circumference values [45]. Although racial differences should be considered, the findings of this and previous studies suggested that we should aggressively perform intervention therapy by exercise training to target the calf circumference as $\geq 34 \mathrm{~cm}$ in males and $\geq 33 \mathrm{~cm}$ in females, resulting in the prevention of adverse cardiovascular events by decline of AVI levels in patients with stage B HFpEF.

\section{Limitations}

This study has several limitations. First, the medical treatments for $\mathrm{HF}$ and/or traditional cardiovascular risk factors could have affected the study results. Second, the calf circumference was measured as a surrogate marker of the muscle mass volume in this study. Nevertheless, calf circumference is a simple parameter of the muscle mass volume. Thus, further studies using accurate methods, such as computed tomography or dual-energy X-ray absorptiometry, are needed to elucidate the correlation between AVI and muscle mass volume. Finally, this was a single-center, cross-sectional study, and the sample size was relatively small. Hence, additional prospective studies, including investigations of interventional therapies, are warranted to elucidate the clinical significance of AVI in patients with stage B HFpEF.

\section{Conclusions}

This study suggests that AVI could be a determining factor for prognosis in patients with stage B HFpEF. Nevertheless, further prospective studies, including intervention therapies, are warranted to validate the results of this study.

\section{Acknowledgments}

The author is grateful to the individuals who participated in this study.

\section{Financial Disclosure}

None to declare.

\section{Conflict of Interest}

None to declare.

\section{Informed Consent}

All patients provided informed consent.

\section{Author Contributions}

The author was involved in preparing the study design as well as acquisition, analysis and interpretation of data.

\section{References}

1. Bhatia RS, Tu JV, Lee DS, Austin PC, Fang J, Haouzi A, Gong Y, et al. Outcome of heart failure with preserved ejection fraction in a population-based study. N Engl J Med. 2006;355(3):260-269.

2. Hernandez AF, Hammill BG, O'Connor CM, Schulman KA, Curtis LH, Fonarow GC. Clinical effectiveness of beta-blockers in heart failure: findings from the OPTIMIZE-HF (Organized Program to Initiate Lifesaving Treatment in Hospitalized Patients with Heart Failure) Registry. J Am Coll Cardiol. 2009;53(2):184-192.

3. Tsuchihashi M, Tsutsui H, Kodama K, Kasagi F, Takeshita A. Clinical characteristics and prognosis of hospitalized patients with congestive heart failure - a study in Fukuoka, Japan. Jpn Circ J. 2000;64(12):953-959.

4. Ruigomez A, Michel A, Martin-Perez M, Garcia Rodriguez LA. Heart failure hospitalization: An important prognostic factor for heart failure re-admission and mortality. Int J Cardiol. 2016;220:855-861.

5. Sueta D, Yamamoto E, Tanaka T, Hirata Y, Sakamoto K, Tsujita K, Kojima S, et al. The accuracy of central blood pressure waveform by novel mathematical transformation of non-invasive measurement. Int $\mathrm{J}$ Cardiol. 2015;189:244-246.

6. Yamanashi H, Koyamatsu J, Nagayoshi M, Shimizu Y, Kawashiri SY, Kondo H, Fukui S, et al. Screening Validity of Arterial Pressure-Volume Index and Arterial Velocity-Pulse Index for Preclinical Atherosclerosis in Japanese Community-Dwelling Adults: the Nagasaki Islands Study. J Atheroscler Thromb. 2018;25(9):792-798.

7. Hitsumoto T. Arterial velocity pulse index as a novel marker of atherosclerosis using pulse wave analysis on high sensitivity troponin $\mathrm{T}$ in hypertensive patients. Cardiol Res. 2017;8(2):36-43.

8. Hitsumoto T. Relationships between the arterial velocity pulse index as a novel marker of atherosclerosis and biomarkers of cardiac or renal condition in patients with type 2 diabetes mellitus. Diabetol Int. 2018;9(1):48-55.

9. Tsao CW, Lyass A, Larson MG, Levy D, Hamburg NM, Vita JA, Benjamin EJ, et al. Relation of Central Arterial Stiffness to Incident Heart Failure in the Community. J 
Am Heart Assoc. 2015;4(11).

10. Hitsumoto T. Efficacy of the reactive oxygen metabolite test as a predictor of initial heart failure hospitalization in elderly patients with chronic heart failure. Cardiol Res. 2018;9(3):153-160.

11. Chow B, Rabkin SW. The relationship between arterial stiffness and heart failure with preserved ejection fraction: a systemic meta-analysis. Heart Fail Rev. 2015;20(3):291-303.

12. Takishima I, Nakamura T, Hirano M, Kitta Y, Kobayashi T, Fujioka D, Saito Y, et al. Predictive value of serial assessment of endothelial function in chronic heart failure. Int J Cardiol. 2012;158(3):417-422.

13. Hunt SA, American College of C, American Heart Association Task Force on Practice G. ACC/AHA 2005 guideline update for the diagnosis and management of chronic heart failure in the adult: a report of the American College of Cardiology/American Heart Association Task Force on Practice Guidelines (Writing Committee to Update the 2001 Guidelines for the Evaluation and Management of Heart Failure). J Am Coll Cardiol. 2005;46(6):e1-82.

14. Sasaki-Nakashima R, Kino T, Chen L, Doi H, Minegishi S, Abe K, Sugano T, et al. Successful prediction of cardiovascular risk by new non-invasive vascular indexes using suprasystolic cuff oscillometric waveform analysis. J Cardiol. 2017;69(1):30-37.

15. Okamoto M, Nakamura F, Musha T, Kobayashi Y. Association between novel arterial stiffness indices and risk factors of cardiovascular disease. BMC Cardiovasc Disord. 2016;16(1):211.

16. Kawakami R, Murakami H, Sanada K, Tanaka N, Sawada SS, Tabata I, Higuchi M, et al. Calf circumference as a surrogate marker of muscle mass for diagnosing sarcopenia in Japanese men and women. Geriatr Gerontol Int. 2015;15(8):969-976.

17. Matthews DR, Hosker JP, Rudenski AS, Naylor BA, Treacher DF, Turner RC. Homeostasis model assessment: insulin resistance and beta-cell function from fasting plasma glucose and insulin concentrations in man. Diabetologia. 1985;28(7):412-419.

18. Imai E, Horio M, Nitta K, Yamagata K, Iseki K, Hara $\mathrm{S}$, Ura N, et al. Estimation of glomerular filtration rate by the MDRD study equation modified for Japanese patients with chronic kidney disease. Clin Exp Nephrol. 2007;11(1):41-50.

19. Mingels A, Jacobs L, Michielsen E, Swaanenburg J, Wodzig W, van Dieijen-Visser M. Reference population and marathon runner sera assessed by highly sensitive cardiac troponin $\mathrm{T}$ and commercial cardiac troponin $\mathrm{T}$ and I assays. Clin Chem. 2009;55(1):101-108.

20. Cesarone MR, Belcaro G, Carratelli M, Cornelli U, De Sanctis MT, Incandela L, Barsotti A, et al. A simple test to monitor oxidative stress. Int Angiol. 1999;18(2):127-130.

21. Schisterman EF, Perkins NJ, Liu A, Bondell H. Optimal cut-point and its corresponding Youden Index to discriminate individuals using pooled blood samples. Epidemiology. 2005;16(1):73-81.

22. Smulyan H, Marchais SJ, Pannier B, Guerin AP, Safar ME, London GM. Influence of body height on pul- satile arterial hemodynamic data. J Am Coll Cardiol. 1998;31(5):1103-1109.

23. Wilkinson IB, MacCallum H, Flint L, Cockcroft JR, Newby DE, Webb DJ. The influence of heart rate on augmentation index and central arterial pressure in humans. J Physiol. 2000;525(Pt 1):263-270.

24. Kim HL, Lim WH, Seo JB, Chung WY, Kim SH, Kim MA, Zo JH. Association between arterial stiffness and left ventricular diastolic function in relation to gender and age. Medicine (Baltimore). 2017;96(1):e5783.

25. Unagami K, Nitta K, Tago K, Matsushita K. Relationship between diastolic dysfunction and atherosclerosis and vascular calcification in hemodialysis patients: diagnostic potential of the cardio-ankle vascular index. Ther Apher Dial. 2016;20(2):135-141.

26. Goto T, Ohte N, Fukuta H, Wakami K, Tani T, Kimura G. Relationship between effective arterial elastance, total vascular resistance, and augmentation index at the ascending aorta and left ventricular diastolic function in older women. Circ J. 2013;77(1):123-129.

27. Takami T, Saito Y. Azelnidipine plus olmesartan versus amlodipine plus olmesartan on arterial stiffness and cardiac function in hypertensive patients: a randomized trial. Drug Des Devel Ther. 2013;7:175-183.

28. Gohar A, Chong JPC, Liew OW, den Ruijter H, de Kleijn DPV, Sim D, Yeo DPS, et al. The prognostic value of highly sensitive cardiac troponin assays for adverse events in men and women with stable heart failure and a preserved vs. reduced ejection fraction. Eur J Heart Fail. 2017;19(12):1638-1647.

29. Ommen SR, Nishimura RA, Appleton CP, Miller FA, Oh JK, Redfield MM, Tajik AJ. Clinical utility of Doppler echocardiography and tissue Doppler imaging in the estimation of left ventricular filling pressures: A comparative simultaneous Doppler-catheterization study. Circulation. 2000;102(15):1788-1794.

30. Saunders JT, Nambi V, de Lemos JA, Chambless LE, Virani SS, Boerwinkle E, Hoogeveen RC, et al. Cardiac troponin $\mathrm{T}$ measured by a highly sensitive assay predicts coronary heart disease, heart failure, and mortality in the Atherosclerosis Risk in Communities Study. Circulation. 2011;123(13):1367-1376.

31. Otaki Y, Arimoto T, Takahashi H, Kadowaki S, Ishigaki D, Narumi T, Honda Y, et al. Prognostic value of myocardial damage markers in patients with chronic heart failure with atrial fibrillation. Intern Med. 2014;53(7):661-668.

32. Deckert T, Feldt-Rasmussen B, Borch-Johnsen K, Jensen T, Kofoed-Enevoldsen A. Albuminuria reflects widespread vascular damage. The Steno hypothesis. Diabetologia. 1989;32(4):219-226.

33. Marechaux S, Samson R, van Belle E, Breyne J, de Monte J, Dedrie C, Chebai N, et al. Vascular and Microvascular Endothelial Function in Heart Failure With Preserved Ejection Fraction. J Card Fail. 2016;22(1):3-11.

34. Meagher P, Adam M, Civitarese R, Bugyei-Twum A, Connelly KA. Heart failure with preserved ejection fraction in diabetes: mechanisms and management. Can J Cardiol. 2018;34(5):632-643.

35. Shah SJ, Lam CSP, Svedlund S, Saraste A, Hage C, Tan 
RS, Beussink-Nelson L, et al. Prevalence and correlates of coronary microvascular dysfunction in heart failure with preserved ejection fraction: PROMIS-HFpEF. Eur Heart J. 2018;39(37):3439-3450.

36. Teo LY, Chan LL, Lam CS. Heart failure with preserved ejection fraction in hypertension. Curr Opin Cardiol. 2016;31(4):410-416.

37. Fazakas A, Szelenyi Z, Szenasi G, Nyiro G, Szabo PM, Patocs A, Tegze N, et al. Genetic predisposition in patients with hypertension and normal ejection fraction to oxidative stress. J Am Soc Hypertens. 2016;10(2):124132.

38. Hitsumoto T. Clinical significance of the augmentation index in patients with preserved kidney function. J Nippon Med Sch. 2012;79(6):422-429.

39. Tanindi A, Erkan AF, Alhan A, Tore HF. Arterial stiffness and central arterial wave reflection are associated with serum uric acid, total bilirubin, and neutrophil-tolymphocyte ratio in patients with coronary artery disease. Anatol J Cardiol. 2015;15(5):396-403.

40. Mahmud A, Feely J. Favourable effects on arterial wave reflection and pulse pressure amplification of adding angiotensin II receptor blockade in resistant hypertension. J Hum Hypertens. 2000;14(9):541-546.
41. Manisty C, Mayet J, Tapp RJ, Sever PS, Poulter N, Mc GTSA, Hughes AD, et al. Atorvastatin treatment is associated with less augmentation of the carotid pressure waveform in hypertension: a substudy of the AngloScandinavian Cardiac Outcome Trial (ASCOT). Hypertension. 2009;54(5):1009-1013.

42. Bekfani T, Pellicori P, Morris DA, Ebner N, Valentova M, Steinbeck L, Wachter R, et al. Sarcopenia in patients with heart failure with preserved ejection fraction: Impact on muscle strength, exercise capacity and quality of life. Int J Cardiol. 2016;222:41-46.

43. Lee SW, Youm Y, Kim CO, Lee WJ, Choi W, Chu SH, Park YR, et al. Association between skeletal muscle mass and radial augmentation index in an elderly Korean population. Arch Gerontol Geriatr. 2014;59(1):49-55.

44. Ohara M, Kohara K, Tabara Y, Igase M, Miki T. Portable indices for sarcopenia are associated with pressure wave reflection and central pulse pressure: the J-SHIPP study. J Hypertens. 2015;33(2):314-322.

45. Gallo LH, Rodrigues EV, Filho JM, da Silva JB, HarrisLove MO, Gomes ARS. Effects of virtual dance exercise on skeletal muscle architecture and function of community dwelling older women. J Musculoskelet Neuronal Interact. 2019;19(1):50-61. 